\title{
Semiconductor-free non-volatile resistive switching memory devices based on metal nanogaps fabricated on flexible substrates via adhesion lithography
}

\author{
James Semple ${ }^{1, *}$, Gwenhivir Wyatt-Moon ${ }^{1}$, Dimitra Georgiadou ${ }^{1}$, Martyn A. McLachlan², Thomas D.
}

Anthopoulos ${ }^{1, *}$

\begin{abstract}
Electronic memory cells are of critical importance in modern-day computing devices, including emerging technology sectors such as large-area printed electronics. One technology that has being receiving significant interest in recent years is resistive switching primarily due to its low-dimensionality and non-volatility. Here, we describe the development of resistive switching memory device arrays based on empty aluminium nanogap electrodes. By employing adhesion-lithography, a lowtemperature and large-area compatible nanogap fabrication technique, dense arrays of memory devices are demonstrated on both rigid and flexible plastic substrates. As prepared devices exhibit non-volatile memory operation with stable endurance, resistance ratios $>10^{4}$ and retention times of several months. Intermittent analysis of the electrode microstructure reveals that controlled resistive switching is due to migration of metal from the electrodes into the nanogap under application of an external electric field. This alternative form of resistive random access memory is promising for use in emerging sectors such as largearea electronics as well as in electronics for harsh environments e.g. space, high/low temperature, magnetic influences, radiation, vibration, pressure.
\end{abstract}

Index Terms - Flexible electronics, nanogap electrodes, nonvolatile memory, resistive switching,

\section{INTRODUCTION}

$\mathrm{E}$ XISTING electronic memory devices typically combine several advantages but also disadvantages. For example, considering memory based on complementary metal oxide semiconductor (CMOS) technology, dynamic random access memory (DRAM) has high capacity and high density but is volatile and must be refreshed every few seconds increasing

Manuscript received October 30, 2016. (Write the date on which you submitted your paper for review.) This work was supported by the European Research Council (ERC) AMPRO grant number 280221, the Engineering and Physical Sciences Research Council (EPSRC) grant number EP/P505550/1 and the EPSRC Centre for Innovative Manufacturing in Large Area Electronics (CIM-LAE) grant no. EP/K03099X/1.

J. Semple, G. Wyatt Moon, D. Georgiadou and T. D. Anthopoulos are with the Centre for Plasic Electronics and Department of Physics at Imperial College London, London SW7 2BZ (+44 (0)20 7594 6669; e-mail: thomas.anthopoulos@imperial.ac.uk/j.semple12@imperial.ac.uk).

M. A. McLachlan is with the Centre for Plastic Electronics and Department of Materials at Imperial College London.

T. D. Anthopoulos is with the Centre for Plasic Electronics, Department of Physics, Imperial College London, London SW7 2AZ, U.K., and also with the Materials Science and Engineering Division of Physical Sciences and Engineering, King Abdullah University of Science and Technology, Thuwal 23955-6900, Saudi Arabia (e-mail: thomas.anthopoulos@imperial.ac.uk). power consumption, while static random access memory (SRAM) is fast but has low capacity and is also volatile. On the other hand, flash memory has high capacity and is nonvolatile, but is characterised by relatively slow operating speed (writing/erasing) [1]. Thus there is a desire to develop new technologies that combine all the advantages of the existing technologies and perhaps offer even more. For example, the ability to demonstrate high-performance memory devices on large-area flexible substrates would be of interest for integration into emerging technologies such as flexible radio frequency identification (RFID) [2]. Amongst emerging technologies, including devices based on ferroelectrics [3], magnetoresistive materials [4], phase-change materials [5] and spin transfer torque memory [6], memristive devices are a prime candidate to fit these requirements. The latter are based on the phenomenon of resistive switching, the effect whereby a solid-state medium exhibits a reversible shift in its bulk resistance on the application of an externally applied electric current or field. The process is often non-volatile and generally observed with materials on the nanoscale, and thus is an interesting avenue of research for potential integration into future technologies.

Broadly speaking, resistive switching occurs by the successive application of electric stress. An initial forming step is required, after which the device enters a low resistance state (LRS). After the forming process, the device in a LRS is switched to a high-resistance state (HRS) by applying a threshold voltage (reset process). Switching back to the LRS (set process) is achieved by applying a threshold voltage that is different to the reset voltage. There is broad discussion on the origin of the switching mechanism, and it varies depending on the material system employed[7]. A popular model is the filament model [8]. In this model, the LRS arises from the formation of a conducting filament through the insulating matrix. Rupture of the filament gives rise to the HRS, but is a reversible process.

Resistive switching is observed in a variety of materials, the majority of research having been carried out on transition metal oxides (TMOs). The first work was carried out in 1960s when Hickmott noticed the effect in a series of binary oxide sandwich structures with different metal electrodes [9]. However, Si based technology took centre stage for several decades until a renewal of interest in the type of switching in the early 2000 was seen as Moore's law called for components on the nanoscale and owing to the advantage of the low power 
consumption of non-volatile devices. Breakthroughs were reported in 2000 by the groups of Ignatiev [10] and Bednorz [11] with reports of reversible and reproducible non-volatile change of the resistance state of two-terminal devices. Since then a range of TMO resistive switching devices have been reported [8], as well as those based on organic polymer systems [12]-[15] and nanomaterials [16]-[18] to name but a few. In tandem with this progress, the emerging field of flexible electronics has evoked interest in new memory technologies compatible with flexible substrates over the past decade, and several investigations into flexible resistive switching devices have been made [19]-[25].

Traditional memristive devices are built in a vertical metalinsulator-metal structure. As visualisation and measurement of conductive pathways in these sandwich type structures has proven difficult, lateral device architectures have emerged in recent years as a tool for understanding filamentation [26][29]. Interestingly, empty lateral electrodes with separation on the nanoscale (nanogap electrodes) have also been shown to exhibit resistive switching behavior [30]-[44]. The concept of using empty nanogap electrodes as switching devices is potentially far more interesting than conventional structures, owing to the simplicity of the system from a fabrication point of view (no insulator/semiconductor material is required) and the possibility of increasing device density. The cause of switching is still debated, being attributed to either conductive filaments forming through the substrate [28], [30]-[33] or changes in the nanogap dimensions leading to different values of tunnel current [34]-[45] .

The apparent bottleneck to the broad adoption of this type of approach however is the difficulty of the fabrication of nanogap electrodes at high throughput and low cost. Nanogap electrode fabrication techniques are traditionally complex and suffer from poor scalability [46]. This has been largely true in the investigation of memristors based on nanogap electrodes, the fabrication techniques employed including electron beam lithography, electromigration and oblique angle evaporation (see Table 1 for full details). Recently, Cui et al. [30] reported the use of atomic layer lithography, a scalable nanogap electrode fabrication technique reported on in recent years [47]-[49], to make arrays of Au nanogap electrodes exhibiting resistive switching effects. While promising, this remains a high temperature fabrication technique, requiring several thermal annealing steps with temperatures up to 500 ${ }^{\circ} \mathrm{C}$, thus rendering it incompatible with flexible substrates. Furthermore, the choice of $\mathrm{Au}$ [34], [39] or Pt [35]-[38] electrodes, commonly used in these studies, may not be a cost effective choice for large scale adoption.

Here, we address these bottlenecks and fabricate, for the first time, nanogap resistive switching devices on flexible substrates. We achieve this by using adhesion lithography (aLith), a large-area and high throughput nanogap electrode patterning technique [50]. Despite its simplicity, a-Lith has proven suitable for the manufacturing of high speed nanoelectronic devices on arbitrary substrates including plastic at high yields [51], [52]. Building on this earlier work, we show that patterning of high aspect ratio aluminium (Al) electrodes in close proximity to each other $(<10 \mathrm{~nm})$ can lead to interesting resistive switching phenomena which can be exploited for the demonstration of non-volatile memory function. The work can be seen as the first step towards a new type of large-area flexible resistive random access memory (RRAM) technology.

\section{EXPERIMENTAL}

Initial tests were carried out on borosilicate glass substrates for ease of handling and initial proof of concept. The process of adhesion lithography for the formation of nanogap electrodes is introduced elsewhere [50]. In brief, Al electrodes $(2 \mathrm{~cm}$ length $\times 3 \mathrm{~mm}$ width $\times 40 \mathrm{~nm}$ height $)$ were patterned via conventional photolithography and wet chemical etching (Fig. 1a, step 1). The samples were then immersed in a $1 \mathrm{mMol}$ solution of octadecylphosphonic acid (ODPA)/proan2-ol (IPA) for 16 hours to allow the formation of a selfassembled monolayer $(\mathrm{SAM})$ on the alumina $\left(\mathrm{AlO}_{\mathrm{X}}\right)$ native to the Al surface, after which they were washed for 30 seconds in IPA and dried with $\mathrm{N}_{2}$ (Fig. 1a, step 2). A ten minute anneal at $80{ }^{\circ} \mathrm{C}$ was employed to ensure complete removal of the solvent. The ODPA was macroscopically observed to attach to the patterned $\mathrm{Al}$ electrode only as when a drop of water was applied, this surface was incredibly hydrophobic, while the substrate remained hydrophilic. A global Al electrode was then deposited via thermal evaporation in high vacuum $\left(10^{-6} \mathrm{mbar}\right)$ (Fig. 1a, step 3). This electrode adheres well to the substrate where no ODPA is present, but poorly to the ODPA coated $\mathrm{AlO}_{\mathrm{X}} / \mathrm{Al}$ surface. On the application and removal of a glue (FirstContact), the poorly adhered Al-on-ODPA is removed, while the strongly adhered Al-on-substrate remains (Fig. 1a, step 4). Fracture occurs at the interfaces, and the result is a nanogap (L) typically on the order of $<10 \mathrm{~nm}$ (Fig. 1b). The ODPA SAM was removed using a 10 minute UV-ozone treatment.

After this, a second photopatterning step is employed to achieve an array of nanogap electrodes with varying width (W) (Fig. 1b). The entire process was repeated on glass with an interlayer of bisbenzocyclobutene (BCB) (Cyclotene 4000, DOW Chemical Company), and on flexible polyimide substrates. Electrical characterisation was carried out using an B2902A Agilent semiconductor parameter analyser under a nitrogen atmosphere at room temperature. Scanning electron micrographs were taken using a LEO Gemini 1525 field emission scanning electron microscope with the operating voltage at $5 \mathrm{kV}$.

\section{FORMING STEP}

Initial current-voltage (I-V) measurements obtained from the as-developed Al-Al nanogap electrodes show no conductivity at moderate biases of $\pm 12 \mathrm{~V}$ (Fig. 2 (a)). This is 
an impressive demonstration of the applicability of a-Lith as a manufacturing process, as there are no shorts despite the channel width being as large as $5 \mathrm{~mm}$ resulting in an aspect ratio for the nanogap on the order of $10^{6}$. At a higher applied bias of $16 \mathrm{~V}$ the device shows increased electrical conductivity (Fig. 2 (b)). However, switching between high and low resistance states is not observed immediately after this step. A further forming step is thus necessary for the nanogap electrode system to enter a resistive switching state. Understanding the nature of the switching is important in determining the best forming step to employ.

Naitoh et al. [53] , who in 2006 first reported resistive switching in empty metal nanogap systems, argued the switching behaviour is not due to the formation of conductive channels, but rather changes in the physical nanogap dimensions. At a low voltage $(+3.5 \mathrm{~V})$ field evaporation with electrostatic attraction or field-induced metal migration were said to be candidates for decrease in nanogap size. A competing repulsive force (either Joule heating or electromigration) was proposed to increase the gap size again. The differences in the HRS and LRS were then supposed to be due to tunneling current at different scales. To this end there is significant evidence that the reported nanogap switching behaviour is due to tunnelling current and a change in gap size, namely the temperature independence of the current, multilevel switching, [53] dependence of switching on the electrode material, [41] and independence of the switching behaviour on substrate material [54]. However, there is also significant evidence that switching can be due to a breakdown in the dielectric substrate (often $\mathrm{SiO}_{2}$ ), such as an independence of switching on electrode material [33], evidence of damage to the substrate [55], and direct imaging of filamentation taking place [28].

With regards to resistive switching in oxide thin films, the forming step is necessary to create the first filaments through the oxide layer. In general a high bias is applied to the system with a compliance current set to prevent complete breakdown [56]. For nanogap electrodes on $\mathrm{SiO}_{2}$, the forming voltage has been found to be $12 \mathrm{~V}$ for a $15 \mathrm{~nm}$ gap [28], $29 \mathrm{~V}$ for a $50 \mathrm{~nm}$ gap [33] and $1.6 \mathrm{~V}$ for $2 \mathrm{~nm}$ gaps [57]. These values would indicate a dielectric strength of $\mathrm{SiO}_{2}$ in the range 6-8 $\mathrm{MV} \mathrm{cm}^{-1}$, a value on par with those reported in the literature [58]. This further implies a breakdown of a-Lith nanogaps, whose separation is on the order of $10 \mathrm{~nm}$, to be on the order of $|10|$ V. However, the applied voltage may need to be higher than this value if there is some voltage drop in the system e.g. due to the presence of a native $\mathrm{Al}_{2} \mathrm{O}_{3}$ layer.

For nanogap dimension change, the forming step is required to decrease the size of the nanogap since its initial dimension is $\sim 10 \mathrm{~nm}$ while tunnelling occurs at length scales $<2 \mathrm{~nm}$. This occurs due to migration or field evaporation of material into the gap which reportedly occurs at field strengths of $10 \mathrm{MV}$ $\mathrm{cm}^{-1}$ [59] . This would correspond to an applied voltage of $\sim 10 \mathrm{~V}$ and has been achieved in other cases where starting nanogap electrodes are on the same scale as those used here by applying a $10 \mathrm{~V}$ pulse [34], [53] or several sweeps between $\pm 10 \mathrm{~V}$ [60]. Thus regardless of the mechanism, the forming step should be fairly similar.

Furthermore, the post-forming $\mathrm{I}-\mathrm{V}$ characteristics are expected to look similar, with a roughly Ohmic current at low voltages and an apparent negative differential resistance (NDR) effect kicking in at somewhere in the 4-6 V range, followed by a flattening out of current. This voltage range tends to be the set voltage, while a higher $(>10 \mathrm{~V})$ value is used as the reset voltage. The only difference in the reported mechanisms is that for nanogap dimension change due to material migration switching, a sweep about the set voltage (e.g. $+10 \mathrm{~V} \rightarrow-1 \mathrm{~V}$, [54] or $+9 \mathrm{~V} \rightarrow+3 \mathrm{~V}$ [53] ) is employed, whereas for oxide switching, a pulse at the set voltage suffices [28], [31], [33], [55], [57].

In activating switching behaviour in $\mathrm{Al}-\mathrm{Al}$ nanogap electrodes fabricated via a-Lith, the use of a forming step based on high voltage sweeps was rarely successful. In some cases, the characteristic NDR effect was observed after dozens of sweeps at or above $\pm 12 \mathrm{~V}$. As an alternative, a fixed voltage was applied for up to 30 seconds while monitoring the current (Fig. 3a). It was found empirically that when the current levels reached $1 \mu \mathrm{A}$, the characteristic NDR peak was observed in IV sweeps (see Fig. 3b). Voltage pulses of increasing current were applied until there was an observed change in current. Values of $14-16 \mathrm{~V}$ were found to be sufficient to observe the desired increase in current. Nanogap electrode pairs of differing widths were investigated, from $2 \mu \mathrm{m}$ up to $5 \mathrm{~mm}$. A successful forming step was seen in devices with widths of 250 $\mu \mathrm{m}$ and above, though not for those of smaller widths. This is believed to be due to as of yet insufficient understanding and optimisation of the forming step, and there should be no physical reason why switching with much smaller device widths is not possible. The shall be further discussed in section V.

\section{SWITCHING CHARACTERISTICS}

In some cases $(<5 \%)$, the application of high bias (16 V) led to a visible destruction of the nanogap interface, thus no switching was observed. In all other cases resistive switching was observed.

Following successful activation the switching characteristics of the nanogap device were investigated. When the device was scanned from high to low voltage potential, it was found to be in a LRS, and remained in this state when voltages below $\sim 3 \mathrm{~V}$ were applied. Current flow of about $20 \mu \mathrm{A}$ was observed at a bias voltage of $2 \mathrm{~V}$, and characteristics were independent of bias direction implying unipolar functionality. Applying higher bias voltages led to the device entering a HRS, where the low voltage current levels were below the detection limits of the measurement setup. It was possible, but not completely reliable, to set the devices (i.e. switch back to the LRS) by applying a voltage pulse in the range $3-8 \mathrm{~V}$. A more reliable method was sweeping from high to low voltage, as reported 
previously for nanogap switching.

To measure the endurance of the devices, a repeated waveform as shown in Fig. 4 was applied to the devices. A 1 ms $12 \mathrm{~V}$ pulse was used as reset voltage and an 8 to $3 \mathrm{~V}$ sweep was used as a set voltage. The current was then measured between 0-2 V. Fig. 5 shows I-V characteristics of a nanogap device in the HRS and LRS in this voltage range. The measured current levels in the LRS are at the detection limit of the setup, implying extracted resistances in the HRS are an underestimation of their true value. The device resistances were extracted at an arbitrary voltage of $0.2 \mathrm{~V}$ and are plotted in Fig. 6. Evidently, the nanogap devices exhibit good endurance over 100 cycles with a measured resistance ratio on the order of $10^{4}$. While certain devices exhibit stable endurance over several hundred cycles, most show some degradation in performance after $\sim 100$ cycles, with device operation showing a greater tendency not to enter the LRS. Interestingly, stopping the scan and running several high voltage I-V sweeps can restore the high quality switching characteristics for a time, implying that with optimisation during the forming step and the set and resest voltage steps, a greater endurance could potentially be achieved. Finally, devices were left in the LRS and HRS and measured after several months showing no change in resistance levels, indicating an extremely good retention time.

\section{SWITCHING MECHANISM}

To examine the cause of the switching behaviour, SEM images of the devices were taken. For reference, Fig. 1b shows the SEM image of an Al-Al electrode nanogap before bias application (as-prepared). After the forming step has been performed, a definite alteration in the electrode conformation is observed. Fig. 8 show representative SEM images of $\mathrm{Al}-\mathrm{Al}$ nanogaps in the LRS (a) and HRS (b) states, respectively, immediately after forming. Clearly there has been severe alteration of the nanogap electrode geometry, especially when compared to the as-prepared device shown in Fig. 1b. In parts the gap has significantly widened, while in others the electrodes remain in close proximity to each other. Closer examination of several SEM micrographs, reveal the presence of small bridging points (a few nanometres across) along the nanogap electrodes in the LRS state (Fig. 8a), which cannot be observed in the electrodes in the HRS state (Fig. 8b). Such metal bridging of electrodes has been observed before, though previously given the presence of a solid electrolyte between the electrodes [61].

These electrical 'bridges' appear to be relatively sparse which may explain the fact that switching behaviour is mainly observed in nanogap electrodes with widths $\geq 250 \mu \mathrm{m}$. In smaller width nanogap electrodes the density of migrated $\mathrm{Al}$ clusters may be too random for reliable switching behaviour to be observed. However, as an exact mechanism for the formation of these bridges is not explored in this study, we posit that the realisation of smaller width and thus higher density nanogap memory devices should in principle be possible.

There is thus evidence to support the migration of $\mathrm{Al}$ into the gap during the forming step, with some points resulting in a breakdown of the electrodes in the vicinity due to heating, and some points approaching each other significantly so as to cause electrical switching. While this switching mechanism is supported by several SEM images, the proof is not definitive and further experimental work will be required to either support or refute the assumed mechanism. As there is much debate on the topic (see for example Table 1), filamentation due to fused Si through the glass substrate could not be ruled out at this stage.

One way to determine which switching mechanism is dominant is to change the substrate material and monitor the impact on the electrical characteristics of the nanogap and the microscopic changes in its geometry. To this end, Al nanogap electrodes were prepared on glass substrates with a $\mathrm{BCB}$ interlayer. This polymer interlayer should be ideal for quenching switching behaviour arising from $\mathrm{SiO}_{2}$ filamentation. While resistive switching in polymers has been reported, this should not be possible in the case of BCB as it has been noted that it is crucial that the polymer be $\pi$ conjugated for switching to arise [62]. Switching is indeed observed on these BCB substrates, with identical characteristics to nanogap devices made on glass substrates.

As a final step, Al-Al nanogap electrodes on polyimide substrates (Fig. 9) were tested for switching behaviour and resultant characteristics mimic both those tested on glass and on BCB coated glass substrates. This gives a strong indication that the switching mechanism is due to the migration of material from the $\mathrm{Al}$ electrodes into the nanogap to form conductive filamentary pathways similar to those shown in Fig. 9a. This is a very interesting finding because, firstly, it demonstrates the compatibility of the nanogap memory technology with plastic substrates, and secondly it represents the first demonstration of a nanogap memristive device on a flexible substrate. Further test must be carried out on the effect of bending on these substrates, where it will be useful to have the device positioned in the substrate centre to directly determine the applied bending radius.

\section{CONCLUSION}

We developed and studied co-planar metal nanogap resistive switching memory devices fabricated using adhesion lithography on arbitrary substrate materials including plastic. We demonstrated that the use of a-Lith enables large-area and low-temperature processing of large nanogap arrays with excellent resistive switching characteristics, the operation of which appears to be independent of the substrate material employed. Using aluminium as the electrode material has led to a further reduction in the cost and fabrication complexity of this rather interesting device concept. As-prepared nanogap devices exhibit robust conductivity switching with resistance 
ratios of $>10^{4}$, excellent endurance and retention times in excess of several months. For successful device operation, an electroforming step based on the application of a $14-16 \mathrm{~V}$ bias for up to 30 seconds was found to be necessary. SEM imaging of the programmed nanogap devices and the independence of the electrical switching behaviour on the substrate material suggest formation of conductive channels due to $\mathrm{Al}$ migration into the nanogap as the cause of switching rather than filamentation through the substrate. An understanding of the specific switching mechanism, as well as the first demonstration of the devices on plastic substrates, is an important stride in the adoption of this interesting technology for non-volatile memory applications on large-area flexible substrates.

\section{REFERENCES}

[1] P. Pavan, R. Bez, P. Olivo, and E. Zanoni, "Flash memory cells-an overview," Proceedings of the IEEE, vol. 85, no. 8, pp. 1248-1271, 1997.

[2] Y.-W. Dai, L. Chen, W. Yang, Q.-Q. Sun, P. Zhou, P.-F. Wang, S.-J. Ding, D. Zhang, and F. Xiao, "Complementary Resistive Switching in Flexible RRAM Devices," Electron Device Letters, IEEE, vol. 35, no. 9, pp. 915-917, 2014.

[3] N. Setter, D. Damjanovic, L. Eng, G. Fox, S. Gevorgian, S. Hong, A. Kingon, H. Kohlstedt, N. Y. Park, G. B. Stephenson, I. Stolitchnov, A. K. Taganstev, D. V. Taylor, T. Yamada, and S. Streiffer, "Ferroelectric thin films: Review of materials, properties, and applications," Journal of Applied Physics, vol. 100, no. 5, p. 051606, 2006.

[4] D. Boeck, V. Roy, Das, Motsnyi, Liu, Lagae, Boeve, Dessein, and Borghs, "Technology and materials issues in semiconductor-based magnetoelectronics," Semiconductor Science and Technology, vol. 17, no. 4, pp. 342-354, 2002.

[5] S. Hudgens and B. Johnson, "Overview of Phase-Change Chalcogenide Nonvolatile Memory Technology," MRS Bulletin, vol. 29, no. 11, pp. 829-832, 2004.

[6] A. Kent and D. Worledge, "A new spin on magnetic memories," Nature nanotechnology, vol. 10, no. 3, pp. 187-191, 2015

[7] D. Jeong, R. Thomas, Katiyar, Scott, Kohlstedt, Petraru, and C. Hwang, "Emerging memories: resistive switching mechanisms and current status," Reports on Progress in Physics, vol. 75, no. 7, p. 076502, 2012.

[8] A. Sawa, "Resistive switching in transition metal oxides," Materials Today, vol. 11, no. 6, pp. 28-36, 2008.

[9] T. W. Hickmott, "Low-Frequency Negative Resistance in Thin Anodic Oxide Films," Journal of Applied Physics, vol. 33, no. 9, p. 2669, 1962.

[10] S. Q. Liu, N. J. Wu, and A. Ignatiev, "Electric-pulse-induced reversible resistance change effect in magnetoresistive films," Applied Physics Letters, vol. 76, no. 19, p. 2749, 2000.

[11] A. Beck, J. G. Bednorz, Ch. Gerber, C. Rossel, and D. Widmer, "Reproducible switching effect in thin oxide films for memory applications," Applied Physics Letters, vol. 77, no. 1, p. 139, 2000.

[12] W.-J. Joo, T.-L. Choi, J. Lee, S. Lee, M.-S. Jung, N. Kim, and J. Kim, "Metal Filament Growth in Electrically Conductive Polymers for Nonvolatile Memory Application," The Journal of Physical Chemistry $B$, vol. 110 , no. 47, pp. 23812-23816, 2006

[13] S. Sivaramakrishnan, P.-J. Chia, Y.-C. Yeo, L.-L. Chua, and P. Ho, "Controlled insulator-to-metal transformation in printable polymer composites with nanometal clusters," Nature Materials, vol. 6, no. 2, pp. 149-155, 2006.

[14] L.-Y. Su and J. Huang, "Demonstration of radio-frequency response of amorphous IGZO thin film transistors on the glass substrate," SolidState Electronics, vol. 104, pp. 122-125, 2015.

[15] L. F. Pender and R. J. Fleming, "Memory switching in glow discharge polymerized thin films," Journal of Applied Physics, vol. 46, no. 8, p. 3426, 1975.
[16] K. S. Vasu, Sampath, and A. K. Sood, "Nonvolatile unipolar resistive switching in ultrathin films of graphene and carbon nanotubes," Solid State Communications, vol. 151, no. 16, pp. 1084-1087, 2011.

[17] S. Porro, E. Accornero, C. Pirri, and C. Ricciardi, "Memristive devices based on graphene oxide," Carbon, vol. 85, pp. 383-396, 2015.

[18] A. Liao, P. Araujo, R. Xu, and M. Dresselhaus, "Carbon nanotube network-silicon oxide non-volatile switches," Nat Commun, vol. 5, p. $5673,2014$.

[19] B. Jang, H. Seong, S. Kim, J. Kim, B. Koo, J. Choi, S. Yang, S. Im, and S.-Y. Choi, "Flexible Nonvolatile Polymer Memory Array on Plastic Substrate via Initiated Chemical Vapor Deposition," ACS Applied Materials \& Interfaces, vol. 8, no. 20, pp. 12951-12958, 2016.

[20] S. Kim, H. Moon, D. Gupta, S. Yoo, and Y.-K. Choi, "Resistive Switching Characteristics of Sol Gel Zinc Oxide Films for Flexible Memory Applications," IEEE T Electron Dev, vol. 56, no. 4, pp. 696699, 2009.

[21] S. Kim and Y.-K. Choi, "Resistive switching of aluminum oxide for flexible memory," Appl Phys Lett, vol. 92, no. 22, p. 223508, 2008.

[22] Y. Cai, J. Tan, L. YeFan, M. Lin, and R. Huang, "A flexible organic resistance memory device for wearable biomedical applications," $P$ Soc Photo-opt Ins, vol. 27, no. 27, p. 275206, 2016.

[23] K. I. Chou, C. H. Cheng, Z. W. Zheng, M. Liu, and A. Chin, "Ni/GeO $/ \mathrm{TiO}_{\mathrm{y}} / \mathrm{TaN}$ RRAM on Flexible Substrate With Excellent Resistance Distribution," IEEE Electr Device L, vol. 34, no. 4, pp. 505507, 2013.

[24] H. Zhao, H. Tu, F. Wei, Z. Shi, Y. Xiong, Y. Zhang, and J. Du, "High mechanical endurance RRAM based on amorphous gadolinium oxide for flexible nonvolatile memory application," J Phys D Appl Phys, vol. 48, no. 20, p. 205104, 2015.

[25] Y. Ji, Y. Yang, S.-K. Lee, G. Ruan, T.-W. Kim, H. Fei, S.-H. Lee, D.-Y Kim, J. Yoon, and J. Tour, "Flexible Nanoporous WO3-xNonvolatile Memory Device," Acs Nano, vol. 10, no. 8, pp. 7598-7603, 2016.

[26] K. Fujiwara, T. Nemoto, M. Rozenberg, Y. Nakamura, and H. Takagi, "Resistance Switching and Formation of a Conductive Bridge in Metal/Binary Oxide/Metal Structure for Memory Devices," Japanese Journal of Applied Physics, vol. 47, no. 8, pp. 6266-6271, 2008.

[27] J. Yang, M. Pickett, X. Li, D. Ohlberg, D. Stewart, and S. Williams, "Memristive switching mechanism for metal/oxide/metal nanodevices," Nature Nanotechnology, vol. 3, no. 7, pp. 429-433, 2008.

[28] J. Yao, L. Zhong, D. Natelson, and J. Tour, "In situ imaging of the conducting filament in a silicon oxide resistive switch," Scientific Reports, vol. 2, p. 242, 2012.

[29] M. Janousch, G. I. Meijer, U. Staub, B. Delley, S. F. Karg, and B. P. Andreasson, "Role of Oxygen Vacancies in Cr-Doped SrTiO3 for Resistance-Change Memory," Advanced Materials, vol. 19, no. 17, pp. 2232-2235, 2007.

[30] A. Cui, Z. Liu, H. Dong, F. Yang, Y. Zhen, W. Li, J. Li, C. Gu, X. Zhang, R. Li, and W. Hu, "Mass Production of Nanogap Electrodes toward Robust Resistive Random Access Memory," Advanced Materials, vol. 28, no. 37, pp. 8227-8233, 2016.

[31] M. Ziegler, O. Harnack, and H. Kohlstedt, "Resistive switching in lateral junctions with nanometer separated electrodes," Solid-State Electronics, vol. 92, pp. 24-27, 2014.

[32] T. Miyabe and T. Nakaoka, "Nanogap Resistance Random Access Memory Based on Natural Aluminum Oxide," Japanese Journal of Applied Physics, vol. 52, no. 4S, p. 04CJ08, 2013.

[33] J. Yao, L. Zhong, Z. Zhang, T. He, Z. Jin, P. Wheeler, D. Natelson, and J. Tour, "Resistive Switching in Nanogap Systems on SiO2 Substrates," Small, vol. 5, no. 24, pp. 2910-2915, 2009.

[34] H. Suga, M. Horikawa, S. Kumaragurubaran, S. Furuta, Y. Masuda, T. Shimizu, and Y. Naitoh, "Resistance switch using metal nanogap electrodes in air," Journal of Applied Physics, vol. 112, no. 4, p. 044309, 2012.

[35] Y. Naitoh, S. Takeshita, D. Ishida, E. Ohmura, K. Kobayashi, H. Yamada, and Y. Majima, "Resistive Switching Effects in Metallic Nanogap Electrode Fabricated by Electroless Gold Plating," Applied Physics Express, vol. 5, no. 8, p. 085201, 2012.

[36] S. Kumaragurubaran, T. Takahashi, Y. Masuda, S. Furuta, T. Sumiya, M. Ono, T. Shimizu, H. Suga, M. Horikawa, and Y. Naitoh, "Nonvolatile high-speed resistance switching nanogap junction memory," Applied Physics Letters, vol. 99, no. 26, p. 263503, 2011. 
[37] H. Suga, M. Horikawa, S. Odaka, H. Miyazaki, K. Tsukagoshi, T. Shimizu, and Y. Naitoh, "Influence of electrode size on resistance switching effect in nanogap junctions," Applied Physics Letters, vol. 97, no. 7, p. $073118,2010$.

[38] H. Suga, T. Sumiya, M. Horikawa, S. Kumaragurubaran, T. Shimizu, and Y. Naitoh, "Threshold Current Density of the Resistance Switching in Pt Nanogap Electrode," Japanese Journal of Applied Physics, vol. 50, no. 6, p. 06GF11, 2011.

[39] Y. Naitoh, H. Suga, and M. Horikawa, "Physical Model for High-toLow Resistive Switching of Gold Nanogap Junction," Japanese Journal of Applied Physics, vol. 50, no. 6, p. 06GF10, 2011

[40] T. Mizukami, Y. Miyato, K. Kobayashi, K. Matsushige, and H. Yamada, "Resistive switching effects in single metallic tunneling junction with nanometer-scale gap," Applied Physics Letters, vol. 98, no. 8 , p. $083120,2011$.

[41] S. Furuta, T. Takahashi, Y. Naitoh, M. Horikawa, T. Shimizu, and M. Ono, "Dependence of Electric Properties of a Nanogap Junction on Electrode Material," Japanese Journal of Applied Physics, vol. 47, no. 3, pp. 1806-1812, 2008.

[42] Y. Naitoh, Y. Morita, M. Horikawa, H. Suga, and T. Shimizu, "NonVolatile Resistance Switching Using Silicon Nanogap Junction," Applied Physics Express, vol. 1, p. 103001, 2008.

[43] K. Takikawa, R. Suda, M. Ito, T. Toyonaka, and J. Shirakashi, "Resistive switching effects in electromigrated Ni nanogaps," 18AD, pp. 715-718.

[44] T. Toyonaka, K. Morihara, K. Takikawa, M. Ito, and J. Shirakashi, "Controlling the tunnel resistance of suspended Ni nanogaps using fieldemission-induced electromigration," Journal of Vacuum Science \& Technology B, vol. 33, no. 2, p. 02B107, 2015.

[45] H. Suga, H. Suzuki, Y. Shinomura, S. Kashiwabara, K. Tsukagoshi, T. Shimizu, and Y. Naitoh, "Highly stable, extremely high-temperature, nonvolatile memory based on resistance switching in polycrystalline $\mathrm{Pt}$ nanogaps," Scientific Reports, vol. 6, p. 34961, 2016.

[46] T. Li, W. Hu, and D. Zhu, "Nanogap Electrodes," Advanced Materials, vol. 22 , no. 2, pp. 286-300.

[47] B. Chen, D. Ji, A. Cheney, N. Zhang, H. Song, X. Zeng, T. Thomay, Q. Gan, and A. Cartwright, "Flat metallic surface gratings with sub-10 nm gaps controlled by atomic-layer deposition," Nanotechnology, vol. 27, no. 37, p. 374003, 2016.

[48] X. Chen, H.-R. Park, M. Pelton, X. Piao, N. Lindquist, H. Im, Y. Kim, J. Ahn, K. Ahn, N. Park, D.-S. Kim, and S.-H. Oh, “Atomic layer lithography of wafer-scale nanogap arrays for extreme confinement of electromagnetic waves," Nature Communications, vol. 4, 2013.

[49] X. Chen, H.-R. Park, N. Lindquist, J. Shaver, M. Pelton, and S.-H. Oh, "Squeezing Millimeter Waves through a Single, Nanometer-wide, Centimeter-long Slit," Scientific Reports, vol. 4, 2014.

[50] D. J. Beesley, J. Semple, L. Jagadamma, A. Amassian, M. A. McLachlan, and T. D. Anthopoulos, "Sub-15-nm patterning of asymmetric metal electrodes and devices by adhesion lithography," Nature communications, vol. 5.

[51] J. Semple, S. Rossbauer, and T. Anthopoulos, "Analysis of Schottky Contact Formation in Coplanar Au/ZnO/Al Nanogap Radio Frequency Diodes Processed from Solution at Low Temperature," ACS Applied Materials \& Interfaces, vol. 8, no. 35, pp. 23167-23174, 2016.

[52] J. Semple, S. Rossbauer, C. Burgess, K. Zhao, L. Jagadamma, A. Amassian, M. McLachlan, and T. Anthopoulos, "Radio Frequency Coplanar ZnO Schottky Nanodiodes Processed from Solution on Plastic Substrates," Small, vol. 12, no. 15, pp. 1993-2000, 2016.

[53] Y. Naitoh, M. Horikawa, H. Abe, and T. Shimizu, "Resistance switch employing a simple metal nanogap junction.," Nanotechnology, vol. 17, no. 22, pp. 5669-74, 2006.

[54] Y. Naitoh, M. Horikawa, and T. Shimizu, "Influence of Substrate Structure on Resistance Switch Using Simple Metal Nanogap Junction," Japanese Journal of Applied Physics, vol. 47, no. 1R, p. 400, 2008.

[55] J. Yao, L. Zhong, D. Natelson, and J. Tour, "Silicon Oxide: A Noninnocent Surface for Molecular Electronics and Nanoelectronics Studies," Journal of the American Chemical Society, vol. 133, no. 4, pp. 941-948, 2011.

[56] X.-J. Zhu, J. Shang, and R.-W. Li, "Resistive switching effects in oxide sandwiched structures," Frontiers of Materials Science, vol. 6, no. 3, pp. 183-206, 2012.
[57] G. Wang, Y. Yang, J.-H. Lee, V. Abramova, H. Fei, G. Ruan, E. Thomas, and J. Tour, "Nanoporous Silicon Oxide Memory," Nano Letters, vol. 14, no. 8, pp. 4694-4699, 2014.

[58] N. Klein and H. Gafni, "The maximum dielectric strength of thin silicon oxide films," Electron Devices, IEEE Transactions on, no. 2, pp. 281289, 1966.

[59] J. Han, K. Song, S. Radhakrishnan, S. Oh, and C. Lee, “A suspended nanogap formed by field-induced atomically sharp tips," Applied Physics Letters, vol. 101, no. 18, p. 183106, 2012.

[60] Y. Naitoh, M. Horikawa, and T. Shimizu, "Effect of Gas Molecules on Resistance Switch Employing a Gold Nanogap Junction," Japanese Journal of Applied Physics, vol. 49, no. 1, p. 01AH08, 2010.

[61] M. Kozicki, M. Park, M. Mitova, "Nanoscale Memory Elements Based on Solid-State Electrolytes," IEEE Transactions on Nanotechnology, vol. 4, p. 331, 2005.

[62] W. Lin, S. Liu, T. Gong, Q. Zhao, and W. Huang, "Polymer- Based Resistive Memory Materials and Devices," Advanced Materials, vol. 26, no. 4, pp. 570-606, 2014. 


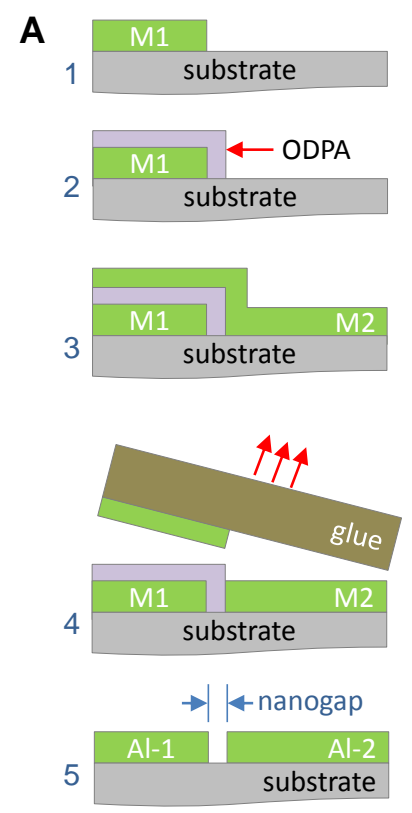

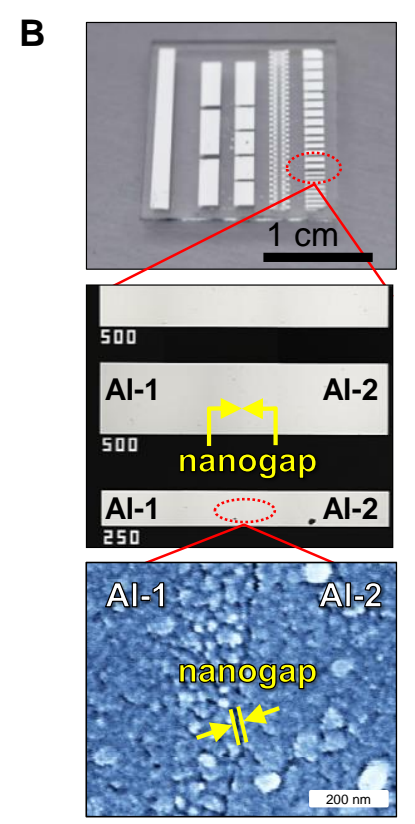

Fig. 1. (a) Schematics of the various pattering steps employed during a-Lith. (b) Aluminium nanogap (NG) electrodes (Al 1 and $\mathrm{Al}$ 2) fabricated via a-Lith on glass substrate with electrode widths ranging from $2 \mu \mathrm{m}$ up to $5,000 \mu \mathrm{m}$. Micrograph images show the array with electrode widths of $250 \mu \mathrm{m}$ and $500 \mu \mathrm{m}$. Scanning electron micrographs show the NG interface between Al 1 and Al 2. Determination of exact gap dimensions is difficult due to resolution limits but it appears to be $<10 \mathrm{~nm}$.
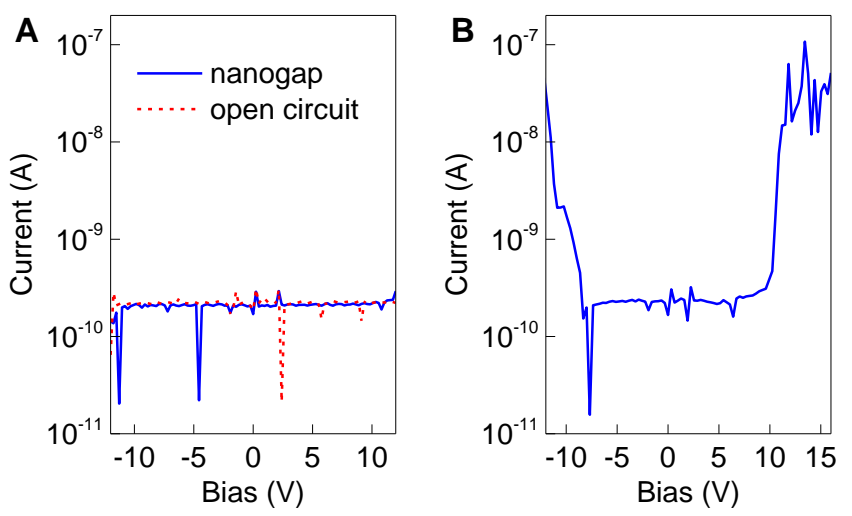

Fig. 2. I-V characteristics of empty nanogap electrodes prior to forming step showing (a) no conductivity up to $12 \mathrm{~V}$ applied bias (an open circuit measurement is shown here also for reference) (b) onset of some current flow with bias of $16 \mathrm{~V}$ but no switching behaviour is observed after the sweep. Small voltage spikes in both cases are due to system noise.
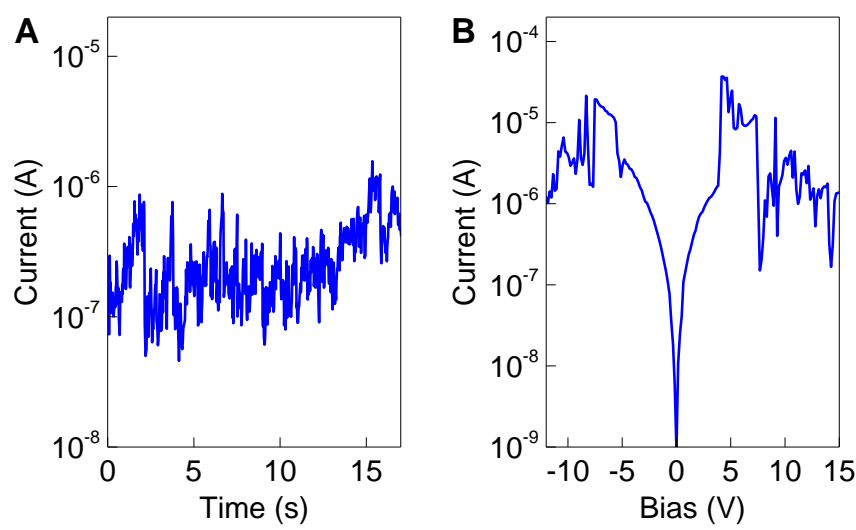

Fig. 3. (a) Forming step for the resistive switching behaviour. Current is plotted vs. time at an applied bias of $14 \mathrm{~V}$. The bias application is stopped after the current exceeds $1 \mu \mathrm{A}$. (b) I-V characteristics of the nanogap electrodes after the forming process showing the expected NDR characteristics.

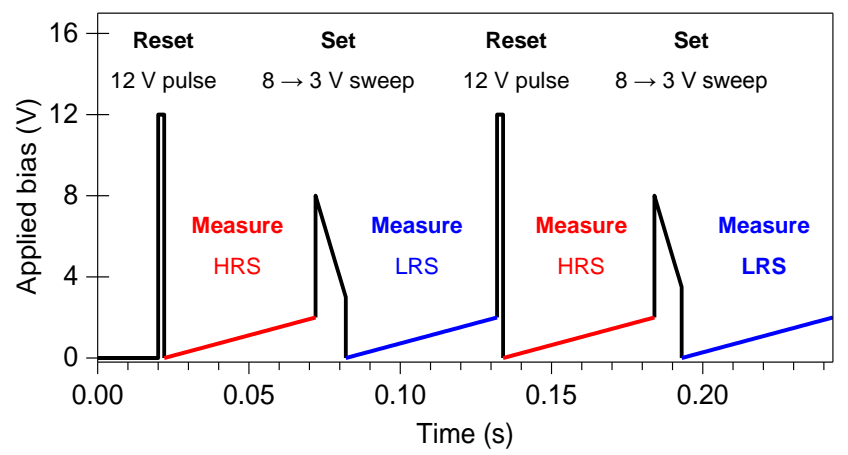

Fig. 4. Applied bias waveform used to evaluate the device endurance. The reset voltage ( $12 \mathrm{~V}$ pulse) applied for $1 \mathrm{~ms}$ puts the device into the HRS, after which the current is measured between $0 \mathrm{~V}$ and $2 \mathrm{~V}$. The set voltage $(8-3 \mathrm{~V}$ sweep) puts the device into the LRS, after which current is again measured in the range between $0 \mathrm{~V}$ and $2 \mathrm{~V}$.

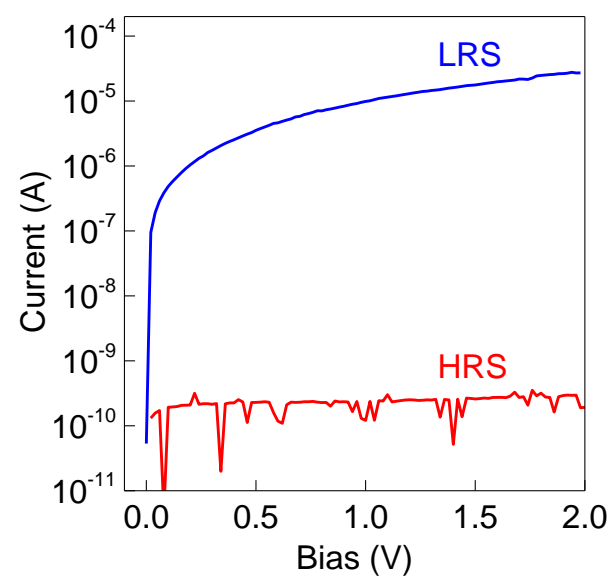

Fig. 5. I-V characteristics of nanogap electrodes in the HRS and LRS using the waveform in Fig. 4 to switch between states and measure. Currents of $\sim 20 \mu \mathrm{A}$ are observed at $2 \mathrm{~V}$ in the LRS. Measured current levels in the HRS are at the detection limit of the measurement setup, implying the real values are lower than those shown here. 


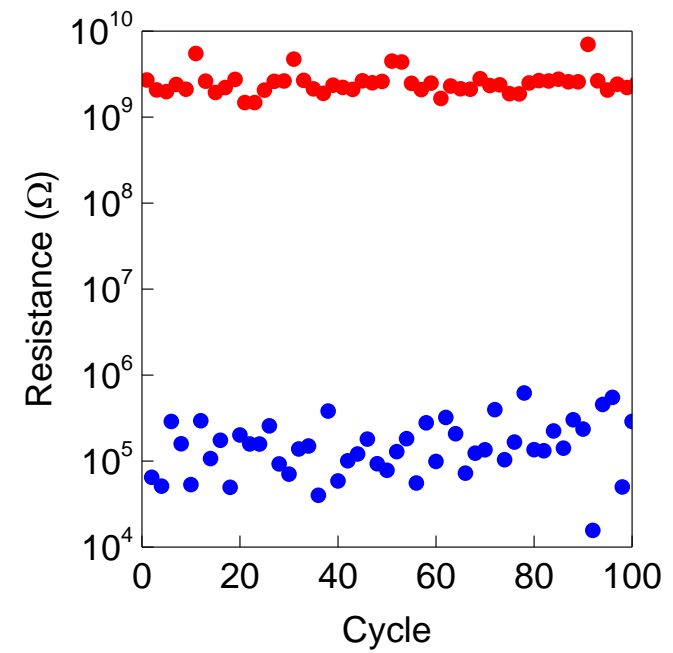

Fig. 6. Endurance of the nanogap electrode memristor over 100 cycles showing uniform repeatability and resistance ratios on the order of $10^{4}$.

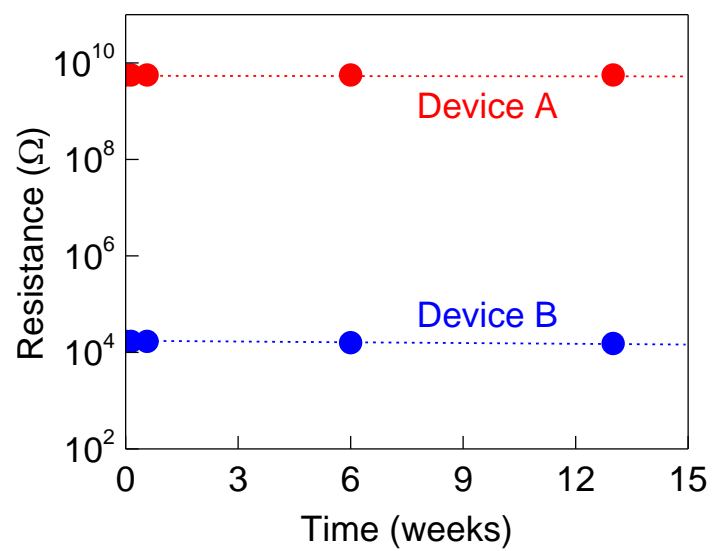

Fig. 7. Retention time for two devices, Device A and Device B held in the HRS and LRS respectively. Both devices show little change in resistance values measured at $2 \mathrm{~V}$ over the course of 13 weeks.
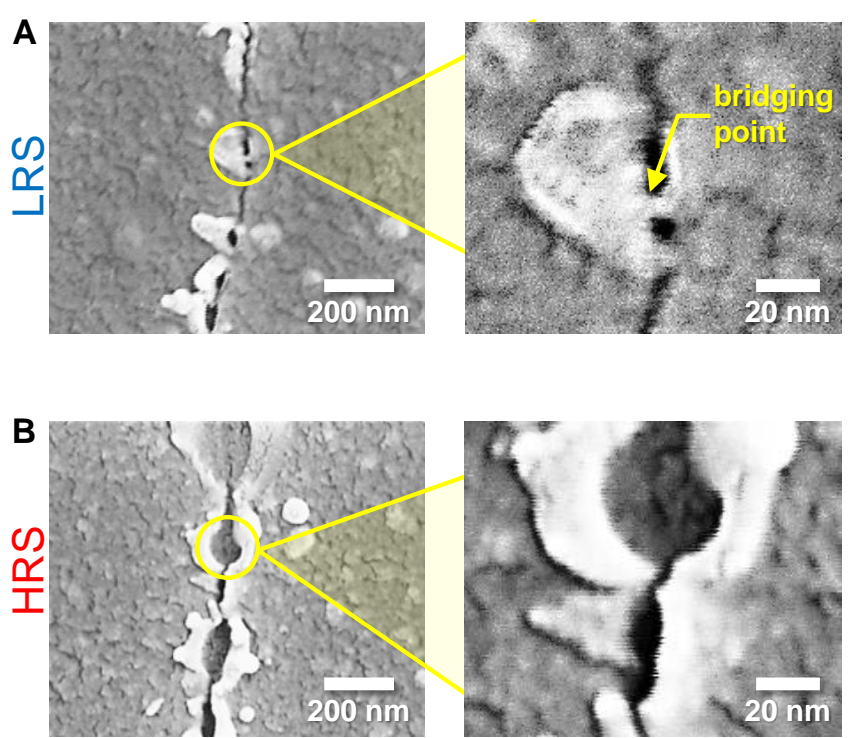

Fig. 8. SEM micrographs of an Al-Al electrode nanogap post forming in the (a) LRS (b) HRS. The higher magnification images reveal evidence of conductive bridges present for devices in the LRS which are absent in the magnified image of the device in the HRS. The images presented here are representative of several dozen images analysed in both the LRS and HRS.

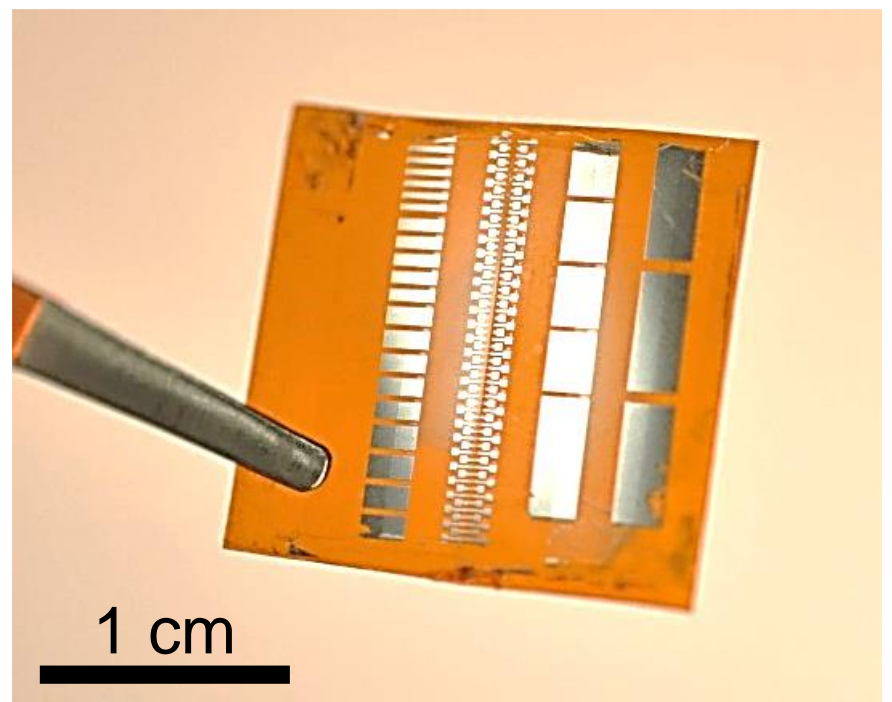

Fig. 9. Array of nanogap electrodes fabricated on flexible polyimide substrate with widths ranging from $2 \mu \mathrm{m}$ up to $5,000 \mu \mathrm{m}$. 
Table 1: Summary of nanogap switching characteristics of devices developed in this work compared to those reported in the literature.

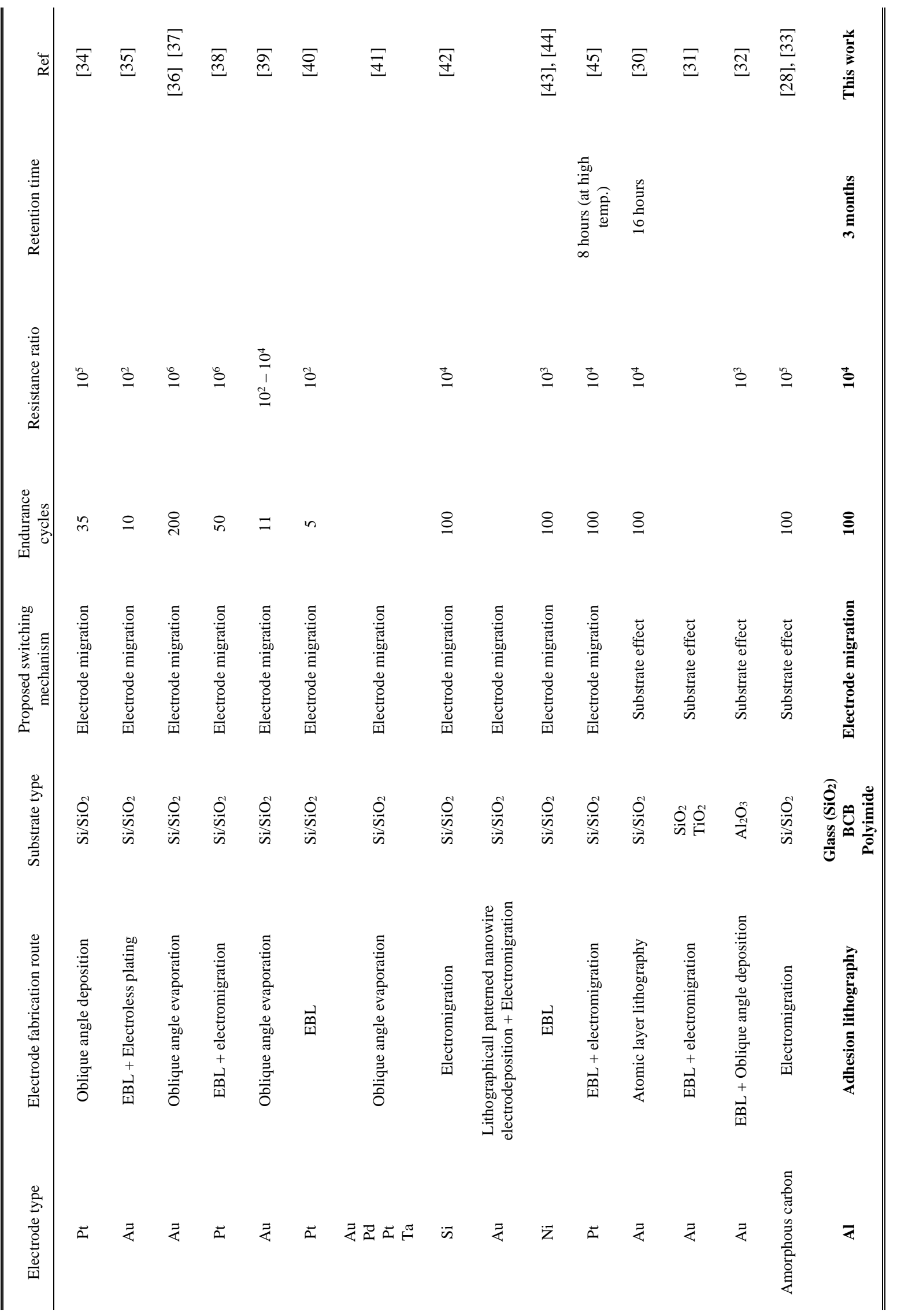


